\title{
Body fluid MMP-2 as a putative biomarker in metastatic breast cancer
}

\author{
SEWON NOH ${ }^{1}$, JAE-JOON JUNG ${ }^{1,4,5}$, MINKYU JUNG ${ }^{1-3}$, KI-HYANG KIM ${ }^{1-3}$, HA-YOUNG LEE ${ }^{1-3}$, \\ BRANDON WANG ${ }^{1}$, JOANNA CHO ${ }^{1}$, TAE SOO KIM ${ }^{1,2}$, HEI-CHEUL JEUNG ${ }^{1-3}$ and SUN YOUNG RHA ${ }^{1-5}$ \\ ${ }^{1}$ Cancer Metastasis Research Center; ${ }^{2}$ Yonsei Cancer Center, Yonsei Cancer Research Institute; \\ ${ }^{3}$ Department of Internal Medicine; ${ }^{4}$ National Biochip Research Center; ${ }^{5}$ Brain Korea 21 Project for Medical Science, \\ Yonsei University College of Medicine, Seoul, Republic of Korea
}

Received July 28, 2011; Accepted December 19, 2011

DOI: $10.3892 / \mathrm{ol} .2012 .549$

\begin{abstract}
In the present study, we investigated the role of matrix metalloproteinase (MMP)-2 and -9 as novel biomarkers in the body fluid of patients with metastatic breast cancer. We measured the expression of MMPs in 37 samples of body fluid (10 peritoneal and 27 pleural fluids) from metastatic breast cancer patients between 2000 and 2009. Zymography and ELISA assays were used to determine the cut-off level and to quantify MMP expression from a positive control, HT-1080 conditioned media. MMP expression in patient samples was measured with ELISA and compared with other clinical parameters. Ascitic carcinoembryonic antigen (CEA) and pleural CEA were measured in patient samples with a chemiluminescent enzyme immunoassay. Body fluid cytology had a positivity of $45 \%(9 / 20)$ for pleural fluid and $28.6 \%$ (2/7) for ascites. However, MMP-2 had a positivity of $85.2 \%(23 / 27)$ in 27 pleural fluid samples and $100 \%$ (10/10) in ascitic fluid with cut-off levels of 8.6 and $0.14 \mathrm{ng} / \mathrm{ml}$ for MMP-2 and -9 , respectively. When body fluid CEA and MMP-2 were combined, the positivity improved to $96 \%$ in pleural fluid and $100 \%$ in ascites. MMP-2 expression in body fluid did not show any significant differences, but MMP-9 expression was lower in ascites than in pleural fluids $(\mathrm{p}<0.005)$. Our results suggest that MMP-2 expression in body fluid be used as an additive diagnostic marker for metastatic breast cancer patients.
\end{abstract}

\section{Introduction}

Breast cancer is one of the most common cancers in the world. There are 2.5 million women diagnosed with breast cancer in the United States, and in Europe. Additionally, 350,000

Correspondence to: Professor Sun Young Rha, Department of Internal Medicine, Division of Medical Oncology, Yonsei University College of Medicine, 250 Seongsanno, Seodaemun-gu, Seoul 120-752, Republic of Korea

E-mail: rha7655@yuhs.ac

Key words: ascites, biomarkers, metastatic breast cancer, matrix metalloproteinase, pleural effusion new cases are diagnosed each year, with a mortality rate of 130,000 patients, accounting for $17.5 \%$ of all cancer-related mortality in Europe $(1,2)$. In Korea, the incidence rate for breast cancer has increased by $2.6 \%$ each year (3). In advanced adenocarcinoma, progressed or stage IV cancer, malignant peritoneal and pleural fluid may develop as the tumor progresses, and this occurs in $10 \%$ of all cases (4).

Clinically, cancer antigen (CA) $15-3$ is widely used as a tumor marker for breast cancer, but it is mostly used with plasma samples. Among the diagnostic methods using body fluid, cytology is thought to be the most reliable, but it is limited by low sensitivity (5). To compensate for the low sensitivity, other diagnostic markers, such as carcinoembryonic antigen (CEA), which has been reported to have a diagnostic value for determining malignancy in pleural fluid, are being used clinically (6). Ascitic CEA has recently been reported to have an increased specificity in peritoneal fluid for diagnosing gastric malignancy (7). However, the markers that are being used to diagnose malignancy still pose problems of low sensitivity with a wide variability, which is a limitation in routine clinical use, particularly for predicting prognosis $(8,9)$.

Matrix metalloproteinases (MMPs) are known to promote cancer progression through extracellular matrix (ECM) and basement membrane degradation, resulting in the exposure of cryptic locations linked to invasion, metastasis and angiogenesis (10-12). It has been reported that active MMPs are indicators for metastasis in breast cancer (13). Additionally, the overexpression of MMP-2 and -9 is reportedly correlated with poor overall survival, suggesting that MMP-2 and -9 are possible prognostic markers $(11,14)$. Therefore, the improved ability to detect malignancy in body fluids of breast cancer patients using biomarkers such as MMPs may be helpful for determining the proper treatment and predicting prognosis. In this study, we evaluated the possibility of using MMP-2 and -9 expressed in body fluids as diagnostic markers for metastatic breast cancer.

\section{Materials and methods}

Patients. We collected the body fluids of 36 patients, who were clinically diagnosed with metastatic stage IV breast carcinoma with malignant ascites or pleural effusion (10 ascites, 
27 pleural fluids; one patient had malignant ascites and pleural effusion) at Yonsei Cancer Center, Yonsei University College of Medicine, Yonsei University Health System between October 2000 and September 2009. Medical records were retrospectively reviewed for patient demographic and clinical information including serum CEA and CA 15-3. The patients had systemic metastasis with more than 2 sites of metastasis, including at least one site of visceral metastasis. The patients were heavily pretreated with systemic chemotherapy, with the median chemotherapy regimen consisting of 3 chemotherapeutic agents (range, 1-7). Clinical and radiological results confirmed that the body fluids originated from the carcinomatosis of breast cancer, with no evidence of other malignancies (15). When the body fluid was detected for the first time in each patient, it was collected through paracentesis or thoracentesis. Body fluid cytology based on cell block and routine body fluid examinations were performed and samples were kept at $-70^{\circ} \mathrm{C}$ until they were used for experimentation. Body fluid cytology results were available in 7 peritoneal and 20 pleural fluids. In addition, CEA expression from body fluids (aCEA for peritoneal and pCEA for pleural fluids) was evaluated with a chemiluminescent enzyme immunoassay (Beckman Coulter Inc., Minnesota, MN, USA). Following the previous result, a positive cut-off level of $5 \mathrm{ng} / \mathrm{ml} \mathrm{CEA} \mathrm{for}$ body fluid was used (7). Patient survival was calculated from the date body fluid was collected until the date of mortality due to any cause. Signed consent was obtained from all patients.

Positivity of body fluid MMP-2 and -9. The cut-off level for positivity of body fluid MMPs determined from a comparison of zymography and ELISA was used based on previous results (16). Briefly, conditioned media (CM) of HT-1080 and human fibrosarcoma cells, were used as a positive control for MMP-2 and -9. To overcome the difficulties of zymography, which shows the qualitative biological activity of MMPs, and also to quantify their activities, an ELISA assay was utilized. Enzymatic activity and the quantitative expression level of MMP were compared with the protein concentration of HT-1080 CM. After confirming the positive correlation between zymography and ELISA results $(\mathrm{p}<0.05)$, we determined the diagnostic cut-off for MMP-9 as $0.14 \mathrm{ng} / \mathrm{ml}$ and $8.6 \mathrm{ng} / \mathrm{ml}$ for MMP-2 (16). Patient samples were then quantified with ELISA assay.

Statistical analysis. To compare the expression levels of body fluid MMPs with body fluid CEA and cytology, the Mann-Whitney U-Test was used. In analyzing the overall survival, we performed a log-rank test using the Kaplan-Meier method. SPSS 13.0 was used to perform all statistical analyses. $\mathrm{P}<0.05$ was considered to indicate a statistically significant difference.

\section{Results}

Biomarker expression in the body fluid of breast cancer patients. Our patient sample included only females $(n=36)$, and the total sample number was 37, as one patient had both malignant ascitic and pleural effusion. The median age of the patients was 54 years (range, 36-77). Body fluid cytology had a $40.7 \%$ (11/27) positivity, serum CA15-3 had a mean value of $159.3 \pm 298.4 \mu \mathrm{g} / \mathrm{ml}$, serum CEA $22.9 \pm 67.9 \mathrm{ng} / \mathrm{ml}$, and body
Table I. Patient characteristics.

\begin{tabular}{lccc}
\hline & Ascites & Pleural effusion & Total \\
\hline $\begin{array}{l}\text { Patient } \\
\text { Female }\end{array}$ & 10 & 27 & 36 \\
$\begin{array}{l}\text { Sample } \\
\text { Body fluid }\end{array}$ & 10 & 27 & 37 \\
$\begin{array}{l}\text { Age, median } \\
\text { (range) }\end{array}$ & $53(40-64)$ & $54(36-77)$ & $54(36-77)$ \\
$\begin{array}{l}\text { Fluid CEA } \\
\text { mean } \pm \text { SD }\end{array}$ & $124.5 \pm 213$ & $37.3 \pm 58.8$ & \\
$\begin{array}{l}\text { Serum CEA } \\
\text { mean } \pm \text { SD }\end{array}$ & $14 \pm 16.6$ & $31.9 \pm 76.5$ & \\
$\begin{array}{l}\text { Serum CA15-3 } \\
\text { mean } \pm \text { SD }\end{array}$ & $380.6 \pm 542.4$ & $88.4 \pm 106.5$ & \\
$\begin{array}{l}\text { Cytology } \\
\text { Positive }\end{array}$ & $2(28.6 \%)$ & $9(45 \%)$ & \\
Negative & $5(71.4 \%)$ & $11(55 \%)$ & \\
$\begin{array}{l}\text { Survival } \\
\text { Median (range) }\end{array}$ & $16(5-792)$ & $291(10-1463)$ & \\
\hline
\end{tabular}

${ }^{\mathrm{a}}$ One patient who has body fluids of ascites and pleural effusion is

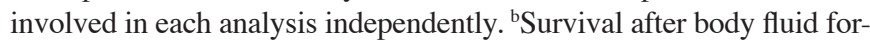
mation. CEA, carcinoembyonic antigen; CA15-3, cancer antigen 15-3.

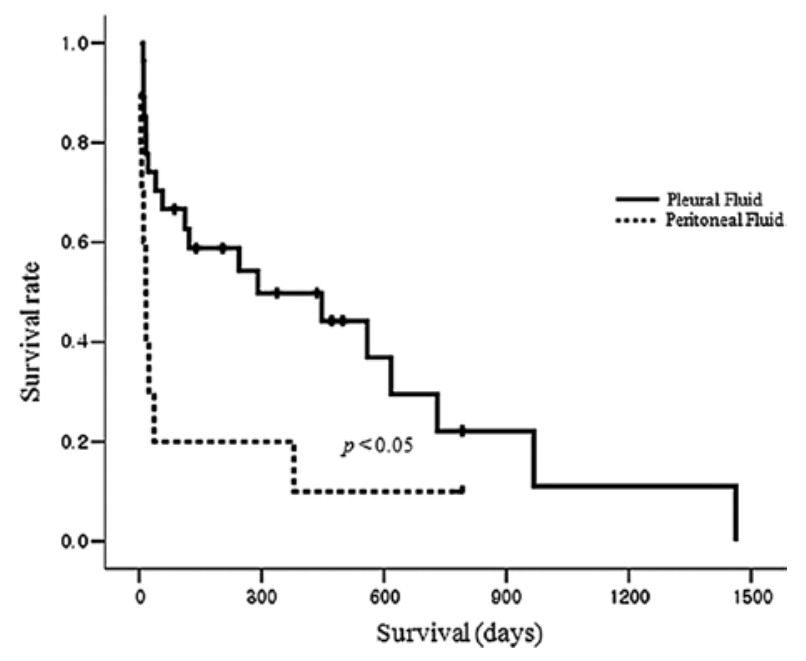

Figure 1. Overall survival compared between body fluids.

fluid CEA had a mean value of $60.9 \pm 124.1 \mathrm{ng} / \mathrm{ml}$ in all patients (Table I). Median overall survival of all patients was 37 days (range, 5-1463), suggesting that the patients had far advanced disease, and patients were heavily pretreated when they developed the malignant ascites of pleural effusion. Notably, our results showed that patients with peritoneal fluid had a significantly shorter survival, with a median of 16 days (range, 5-792), compared to patients with pleural fluid, who had a median survival of 291 days (range, 10-1463), p<0.05 (Fig. 1).

Since body fluid CEA has been reported to have a role as a biomarker (7), we evaluated CEA in body fluids. CEA 
Table II. Comparison of body fluid biomarker expression between ascites and pleural effusion.

\begin{tabular}{lcclc}
\hline & N & MMP-9 & \multicolumn{1}{c}{ MMP-2 } & CEA \\
\hline Ascites & 10 & $0.09 \pm 0.26$ & $34.1 \pm 20$ & $124.5 \pm 213$ \\
Pleural effusion & 27 & $0.25 \pm 0.64$ & $29.9 \pm 24.5$ & $37.3 \pm 58.8$ \\
Total & 37 & $0.20 \pm 0.56$ & $31.1 \pm 23.19$ & $60.9 \pm 124.1$
\end{tabular}

MMP-9, matrix metalloproteinase-9; MMP-2, matrix metalloproteinase-2; CEA, carcinoembyonic antigen.

expression differed in body fluids; ascites had a higher CEA expression of $124.5 \pm 213 \mathrm{ng} / \mathrm{ml}$ than the CEA expression in pleural fluids, which was $37.3 \pm 58.8 \mathrm{ng} / \mathrm{ml}$. We then compared MMP-9 and -2 expression in body fluids. Peritoneal fluid had a lower MMP-9 expression level of $0.09 \pm 0.26 \mathrm{ng} / \mathrm{ml}$ compared to $0.25 \pm 0.64 \mathrm{ng} / \mathrm{ml}$ from the pleural fluids. However, MMP-2 expression in ascites $(34.1 \pm 20 \mathrm{ng} / \mathrm{ml})$ was higher than that in pleural fluids $(29.9 \pm 24.5 \mathrm{ng} / \mathrm{ml}$ ) (Table II). When we compared the expression levels of various biomarkers (CEA, MMP-2, MMP-9) in ascites and pleural fluids, CEA and MMP-2 were not significantly different (data not shown). In comparison, MMP-9 expression in pleural fluid was higher than that in peritoneal fluid $(\mathrm{p}<0.05)$.

Improved malignancy detection using body fluid MMP-2. Cytology information from 20 pleural and 7 peritoneal fluids showed positive rates of $45(9 / 20)$ and $28.6 \%$ (2/7), respectively, demonstrating that cytology has an overall low sensitivity in our samples, as reported in previous studies $(5,9)$. Following evaluation of other biomarkers in 27 pleural fluids, MMP-2 was found to have the highest positivity with $85.2 \%$ (23/27), followed by CEA, with a $74.1 \%$ (20/27) positivity. MMP-9 showed the lowest positivity with $29.6 \%$ (8/27) (Table III). Notably, in five patients with ascites and negative cytology from peritoneal fluid, four patients were positive for CEA expression (80\%), one patient was positive for MMP-9 expression (20\%), and all five patients were positive for MMP-2 expression (100\%). By contrast, in 11 pleural fluids with negative cytology, the positivity for CEA, MMP-2 and MMP-9 were $63.6(7 / 11)$, 72.7 (8/11) and 27.3\% (3/11), respectively. These results suggest that body fluid MMPs, especially MMP-2, could be used as diagnostic biomarkers in metastatic breast cancer.

When the biomarkers were combined, an increase in sensitivity was observed. In pleural fluids, combining CEA and MMP-2 increased the positivity to $96.3 \%$ (26/27). Combining MMP-9 and MMP-2 showed a positive rate of 88.9\% (24/27), combining CEA and MMP-9 improved the positivity to $85.2 \%$ (23/27), and combining all three markers had the same positive rate as combining just CEA and MMP-2, 96.3\% (26/27) (Table III). In the 10 peritoneal fluid samples, MMP-2 had the highest positive rate with 100\% (10/10), followed by CEA which had a positivity of $80 \%(8 / 10)$, and MMP-9 had the lowest positive rate with $10 \%(1 / 10)$. The combination of body fluid CEA and body fluid MMP-2 had a positive rate of $100 \%$ (10/10), which was equal to combining body fluid MMP-9
Table III. Positivity of single and multiple biomarkers in body fluids.

\begin{tabular}{|c|c|c|c|c|}
\hline & \multicolumn{2}{|c|}{$\begin{array}{c}\text { Ascites } \\
(\text { Total } n=10)\end{array}$} & \multicolumn{2}{|c|}{$\begin{array}{l}\text { Pleural fluids } \\
\text { (Total } n=27 \text { ) }\end{array}$} \\
\hline & $\mathrm{n}$ & $\%$ & $\mathrm{n}$ & $\%$ \\
\hline \multicolumn{5}{|l|}{ Single marker } \\
\hline CEA & 8 & 80 & 20 & 74.1 \\
\hline MMP-9 & 1 & 10 & 8 & 29.6 \\
\hline MMP-2 & 10 & 100 & 23 & 85.2 \\
\hline \multicolumn{5}{|l|}{ Multiple markers } \\
\hline CEA+MMP-9 & 8 & 80 & 23 & 85.2 \\
\hline CEA+MMP-2 & 10 & 100 & 26 & 96.3 \\
\hline MMP-9+MMP-2 & 10 & 100 & 24 & 88.9 \\
\hline CEA+MMP-9+MMP-2 & 10 & 100 & 26 & 96.3 \\
\hline
\end{tabular}

CEA, carcinoembryonic antigen; MMP-9, matrix metalloproteinase-9; MMP-2, matrix metalloproteinase-2.

and body fluid MMP-2 or combining all three biomarkers. The combination of body fluid CEA and body fluid MMP-9, however, increased positivity to $80 \%(8 / 10)$ (Table III). As a result, MMP-2 was more sensitive to detecting malignancy in body fluids and had an additional diagnostic role when combined with body fluid CEA.

Previous reports have suggested that body fluid CEA is a marker with a relatively high sensitivity of approximately $80 \%$ in the body fluid of various types of cancer, confirming results of this study, obtained from the body fluid of metastatic breast cancer patients $(6,7,16)$. However, our results showed that body fluid MMP-2 had an even higher sensitivity than body fluid CEA for detecting malignancy in breast carcinoma. In pleural fluids, the combination of body fluid MMP-2 and body fluid CEA improved sensitivity to almost $100 \%$, indicating that MMP-2 alone, or in addition to CEA, may be used as a diagnostic biomarker. In peritoneal fluid, MMP-2 had a positivity of $100 \%$, suggesting that body fluid MMP-2 is a useful diagnostic biomarker in metastatic breast cancer patients, in addition to cytology and body fluid CEA.

\section{Discussion}

We evaluated the expression of MMP-2 and -9 in the body fluid of metastatic breast cancer patients to determine the possibility of using MMPs as biomarkers. MMPs are reportedly involved in prognosis and are used as prognostic markers in tissue and plasma samples $(17,18)$. Our study focused on body fluid samples, which have an advantage over tissue and plasma samples in terms of their availability and representation of the direct effect from cancer. Body fluids may be obtained from patients as soon as the fluids accumulate, but tissue samples are limited in their availability. Moreover, plasma samples are not directly in contact with the cancer, and thus may contain numerous non-specific target molecules, whereas body fluids form directly at the site of cancer and are capable of reflecting cancer status as well as tumor burden. Therefore, body 
fluids provide advantages over tissue and plasma samples for understanding pathogenesis and diagnosis, and for predicting clinical outcomes in breast cancer.

Cytology from body fluid is known to be the most reliable marker for diagnosis, but has an extremely low sensitivity (5). Due to the low sensitivity, other cancer-related biomarkers such as CEA and telomerase activity are being used in patient samples, but still yield unsatisfactory sensitivities and specificities, and are not applicable due to difficulties in detection methods. We aimed to identify diagnostic biomarkers with increased sensitivity by evaluating MMP-2 and -9 expression in body fluids to be used alone or in addition to body fluid CEA, for detecting malignancy within body fluids from metastatic breast cancer.

Since our study focused on assessing body fluid MMP-2 and -9 as diagnostic markers in breast cancer, the choice of method for evaluation was important, as the assay directly affects accuracy and practicality. While zymography allows visualization of the enzymatic activity of MMPs qualitatively, ELISA provides a quantitative amount of MMP protein expression. The combination of methods allows us to create an accurate assessment of MMP expression (19). Using the two assays with HT-1080 CM, we determined a cut-off level for MMP-9 of 0.14 and $8.6 \mathrm{ng} / \mathrm{ml}$ for MMP-2 based on the minimal level of expression that could be sufficient for diagnosis.

In our experiment, body fluid MMP-2 had a positivity of $85.2 \%$ in pleural fluid and $100 \%$ in peritoneal fluid. When body fluid MMP-2 was combined with body fluid CEA, positivity was increased to $96.3 \%$ in pleural fluid. Compared to body fluid MMP-9 with limited diagnostic features, body fluid MMP-2 alone or in combination with body fluid CEA was useful as an additive diagnostic marker in the body fluid of breast cancer patients. Although body fluid MMPs did not seem to have any prognostic role in our study (data not shown), the type of body fluid had a prognostic role. Breast cancer patients with peritoneal fluid had a significantly shorter survival compared to patients with pleural fluid. This observation may be correlated with disease burden considering the site of metastasis from the original breast tumor. Previously, it was reported that MMP-2 and MMP-9 are involved in breast cancer invasion $(20,21)$. Our results have shown that MMP-2 has a higher expression and positivity than MMP-9. Findings of a previous report that evaluated 23 malignant body fluids showed that MMP-2 (87\%) had a higher positivity than MMP-9 $(78.3 \%)$ in different cancer origins, corresponding with our result (22). In addition, MMP-9 expression was significantly higher in pleural than in peritoneal fluid. Breast cancer patients initially form pleural fluid, which may invade to cause the formation of peritoneal fluid in the abdomen. Since MMP-2 and -9 are involved in cancer invasion, MMP expression may be higher in pleural fluid in preparation for cancer invasion, whereas MMP expression may be lower in peritoneal fluid, suggesting that invasion has already occurred. Studies have shown that MMP-9 is capable of being downregulated after invasion and body fluid formation has occurred, suggesting that MMP-9 expression is tightly controlled, which may contribute to the low level of MMP-9 in peritoneal fluid $(23,24)$. In one patient who had both ascitic fluid and pleural effusion, MMP-9 expression in the pleural fluid $(0.55 \mathrm{ng} / \mathrm{ml})$ was much greater than that in the ascitic fluid $(0.02 \mathrm{ng} / \mathrm{ml})$.
Previous reports determined that cancer invasion is correlated with poor survival (25). We observed that the patients with malignant ascites showed a shorter survival compared to the patients with pleural effusion. As pleural and peritoneal effusions occur in various parts of the body with different biology, we consider that the body fluid itself may differ in the expression and biological role of each molecule, which may also predict prognosis. Previous reports have suggested that MMP-9 and MMP-2 overexpression correlates with poor overall survival in tissue and plasma samples of various cancers $(17,18)$. However, results from our experiment did not concur with these reports, except for the prognostic potential of the site of malignant effusion. This may be related to the small sample size in our study. Moreover, since our study used body fluid, rather than tissue and plasma, the expression of MMPs may differ, since MMPs are under tight control in the body and in cells $(23,24)$.

Although we focused on the role of body fluid biomarkers, if we could use the serum biomarkers in addition to the body fluid biomarkers, more reliable information would be obtained in order to understand the status of the patients, including tumor burdens and prognosis. Among the numerous tumor markers, serum CEA and CA 15-3 are mostly used for breast cancer patients. However, in our patient set, the level of those serum markers were not correlated with each other or with body fluid biomarkers. In addition, pleural CEA has been used for the detection of malignancy from pleural fluid. However, ascitic CEA has recently been suggested as a detection factor for malignancy by our previous report in gastric cancer. Therefore, in this study we compared ascitic and pleural CEA with body fluid MMPs. Our study was unique in that it analyzed MMP expression in body fluids of metastatic breast cancer patients, allowing us to compare the differences that potentially exist between each patient. Moreover, the use of an ELISA assay has several benefits for use in clinical practice; it is easy, quantitative and a small amount of the body fluid is required. Previous studies have suggested that MMP-2 and -9 be used as diagnostic markers in tissue and plasma samples. However, this is the first study to use malignant body fluids from breast cancer to evaluate MMPs as possible diagnostic markers. In particular, our results suggest that MMP-2 is a highly sensitive diagnostic marker for metastatic breast cancer patients. The limitations of the current study are: i) the patient heterogeneity, ii) the study is retrospective with a small sample size, and iii) the determination of the assay cut-off level is arbitrary. Patient heterogeneity with tumor heterogeneity is the essential problem of translational research. In our study, patients with relatively homogeneous clinical features were selected. The patients were required to have clinically evident malignant ascites or pleural effusion regardless of cytology results, considering the false negativity of body fluid cytology. Since systemic chemotherapy after body fluid formation may affect prognosis, we selected patients who had received active systemic chemotherapy prior to body fluid formation. Therefore, patients who developed ascites or pleural effusion at the time of breast cancer diagnosis were excluded. Multiple sites of metastasis were also observed, including a minimum of 1 visceral metastasis from the breast cancer. All the patients were previously heavily pretreated with active systemic treatment. In addition, this study is the first study to evaluate the 
proof-of-concept of whether the biomarker in the body fluid may work in clinical practice and also the feasibility of ELISA for stratifying the patients. In almost all the patients who develop body fluids, the fluid examination is easily performed in clinical practice, and the collection of body fluid is feasible. Gathering the fluid provides biological information, which may be of clinical use, and therefore this practice is worthwhile. However, validation with large numbers of prospectively collected samples is required for the further clinical development of these novel body fluid biomarkers.

\section{Acknowledgements}

This manuscript was supported by the Public Welfare and Safety research program through the National Research Foundation of Korea (NRF) funded by the Ministry of Education, Science and Technology (2010-0020841).

\section{References}

1. Tyczynski JE, Plesko I, Aareleid T, et al: Breast cancer mortality patterns and time trends in 10 new EU member states: mortality declining in young women, but still increasing in the elderly. Int J Cancer 112: 1056-1064, 2004.

2. Holmes MD, Chen WY, Hankinson SE and Willett WC: Physical activity's impact on the association of fat and fiber intake with survival after breast cancer. Am J Epidemiol 170: 1250-1256, 2009.

3. Jung KW, Won YJ, Park S, et al: Cancer statistics in Korea: incidence, mortality and survival in 2005. J Korean Med Sci 24 995-1003, 2009

4. Runyon BA, Hoefs JC and Morgan TR: Ascitic fluid analysis in malignancy-related ascites. Hepatology 8: 1104-1109, 1988.

5. Abe $\mathrm{S}$, Yoshimura $\mathrm{H}$, Tabara $\mathrm{H}$, et al: Curative resection of gastric cancer: limitation of peritoneal lavage cytology in predicting the outcome. J Surg Oncol 59: 226-229, 1995.

6. Terracciano D, Di Carlo A, Papa P, et al: New approaches in the diagnostic procedure of malignant pleural effusions. Oncol Rep 12: 79-83, 2004.

7. Jung M, Jeung HC, Lee SS, et al: The clinical significance of ascitic fluid CEA in advanced gastric cancer with ascites. J Cancer Res Clin Oncol 136: 517-526, 2010.

8. Gaspar MJ, Arribas I, Coca MC and Diez-Alonso M: Prognostic value of carcinoembryonic antigen, CA 19-9 and CA 72-4 in gastric carcinoma. Tumour Biol 22: 318-322, 2001.

9. de Manzoni G, Verlato G, Di Leo A, et al: Peritoneal cytology does not increase the prognostic information provided by TNM in gastric cancer. World J Surg 30: 579-584, 2006.
10. Egeblad M and Werb Z: New functions for the matrix metalloproteinases in cancer progression. Nat Rev Cancer 2: 161-174, 2002.

11. Duffy MJ, Maguire TM, Hill A, McDermott E and O'Higgins N: Metalloproteinases: role in breast carcinogenesis, invasion and metastasis. Breast Cancer Res 2: 252-257, 2000.

12. Curran S and Murray GI: Matrix metalloproteinases: molecular aspects of their roles in tumour invasion and metastasis. Eur J Cancer 36: 1621-1630, 2000.

13. Jezierska A and Motyl T: Matrix metalloproteinase-2 involvement in breast cancer progression: a mini-review. Med Sci Monit 15: RA32-40, 2009.

14. Dragutinovic V, Izrael-Zivkovic L and Radovanovic N: Relation of matrix metalloproteinase-9 to different stages of tumors in the serum of gastric cancer. Dig Dis Sci 54: 1203-1207, 2009.

15. Yajima K, Kanda T, Ohashi M, et al: Clinical and diagnostic significance of preoperative computed tomography findings of ascites in patients with advanced gastric cancer. Am J Surg 192: 185-190, 2006.

16. Noh S, Jung JJ, Jung M, et al: MMP-2 as a putative biomarker for carcinomatosis in gastric cancer. Hepatogastroenterology 58: 2011 (Epub ahead of print).

17. Lengyel E, Schmalfeldt B, Konik E, et al: Expression of latent matrix metalloproteinase 9 (MMP-9) predicts survival in advanced ovarian cancer. Gynecol Oncol 82: 291-298, 2001.

18. Kallakury BV, Karikehalli S, Haholu A, Sheehan CE, Azumi N and Ross JS: Increased expression of matrix metalloproteinases 2 and 9 and tissue inhibitors of metalloproteinases 1 and 2 correlate with poor prognostic variables in renal cell carcinoma. Clin Cancer Res 7: 3113-3119, 2001.

19. Lombard C, Saulnier J and Wallach J: Assays of matrix metalloproteinases (MMPs) activities: a review. Biochimie 87: 265-272, 2005.

20. Shah FD, Shukla SN, Shah PM, Shukla HK and Patel PS: Clinical significance of matrix metalloproteinase 2 and 9 in breast cancer. Indian J Cancer 46: 194-202, 2009.

21. Hong MK, Cho KY, Oh SJ, Kim KM, Yu SJ and Jung SS: Implications of the Activation of Matrix Metalloproteinase-2 (MMP-2) on the Metastasis in Breast Cancer. J Korean Breast Cancer Soc 5: 19-26, 2002.

22. Sun XM, Dong WG, Yu BP, Luo HS and Yu JP: Detection of type IV collagenase activity in malignant ascites. World $\mathrm{J}$ Gastroenterol 9: 2592-2595, 2003.

23. McCawley LJ and Matrisian LM: Matrix metalloproteinases: multifunctional contributors to tumor progression. Mol Med Today 6: 149-156, 2000.

24. Belotti D, Paganoni P, Manenti L, et al: Matrix metalloproteinases (MMP9 and MMP2) induce the release of vascular endothelial growth factor (VEGF) by ovarian carcinoma cells: implications for ascites formation. Cancer Res 63: 5224-5229, 2003.

25. Warren M: Metastatic breast cancer recurrence: a literature review of themes and issues arising from diagnosis. Int J Palliat Nurs 15: 222-225, 2009 